\title{
Publisher's Note: Evidence for various higher-subband resonances and interferences in a GaAs/AlAs asymmetric quadruple-quantum-well superlattice analyzed from its photoluminescence properties [Phys. Rev. B 95, 075309 (2017)]
}

Keisuke Hata, Makoto Hosoda, Kouichi Akahane, and Naoki Ohtani (Received 23 February 2017; published 28 February 2017)

DOI: 10.1103/PhysRevB.95.079907

This paper was published online on 22 February 2017 with an error in Fig. 8. Figure 8 has been replaced as of 24 February 2017. The figure is correct in the printed version of the journal. 